\title{
Algebraic independence of Fredholm series
}

\author{
by \\ Kumiko Nishioka (Yokohama)
}

1. Introduction. Let $K$ be an algebraic number field and $d \geq 2$ be an integer. We call

$$
f(z)=\sum_{h=0}^{\infty} \sigma_{h} z^{d^{h}}, \quad \sigma_{h} \in K^{\times}, \log \left\|\sigma_{h}\right\|=o\left(d^{h}\right),
$$

a Fredholm series. The convergence radius of $f(z)$ is 1 . By Hadamard's gap theorem, the unit circle is the natural boundary of $f(z)$. If $\alpha$ is an algebraic number with $0<|\alpha|<1$, then $f(\alpha)$ is transcendental (cf. Theorem 2.10.1 in Nishioka [2]). Let

$$
f_{d}(z)=\sum_{h=0}^{\infty} \sigma_{d h} z^{d^{h}}, \quad \sigma_{d h} \in K^{\times}, \log \left\|\sigma_{d h}\right\|=o\left(d^{h}\right), d=2,3, \ldots
$$

Then we may expect that $f_{d}(\alpha), d=2,3, \ldots$, are algebraically independent. When $\sigma_{d h}=1$ for all $d, h$, this is proved in Nishioka [3]. Here we will prove the following.

THEOREM 1. If for every $d$, the $\sigma_{d h}(h=0,1, \ldots)$ are in a finite set of nonzero algebraic numbers, then $f_{d}(\alpha), d=2,3, \ldots$, are algebraically independent for any algebraic number $\alpha$ with $0<|\alpha|<1$.

2. Mahler's method. By $\mathbb{N}$ and $\mathbb{N}_{0}$ we denote the set of positive integers and the set of nonnegative integers respectively. If $\alpha$ is an algebraic number, we denote by $\alpha$ the maximum of the absolute values of the conjugates of $\alpha$ and by $\operatorname{den}(\alpha)$ the least positive integer such that $\operatorname{den}(\alpha) \alpha$ is an algebraic integer, and we set $\|\alpha\|=\max \{\alpha, \operatorname{den}(\alpha)\}$. Then we have the inequalities

$$
|\alpha| \geq\|\alpha\|^{-2[\mathbb{Q}(\alpha): \mathbb{Q}]} \quad \text { and } \quad\left\|\alpha^{-1}\right\| \leq\|\alpha\|^{2[\mathbb{Q}(\alpha): \mathbb{Q}]}
$$

2000 Mathematics Subject Classification: Primary 11J81.

Key words and phrases: algebraic independence, Mahler's method, Fredholm series. 
(cf. Lemma 2.10.2 in [2]). If $\Omega=\left(\omega_{i j}\right)$ is an $n \times n$ matrix with nonnegative integer entries and $z=\left(z_{1}, \ldots, z_{n}\right)$ is a point of $\mathbb{C}^{n}$, we define a transformation $\Omega: \mathbb{C}^{n} \rightarrow \mathbb{C}^{n}$ by

$$
\Omega z=\left(\prod_{j=1}^{n} z_{j}^{\omega_{1 j}}, \ldots, \prod_{j=1}^{n} z_{j}^{\omega_{n j}}\right) .
$$

Let $\left\{\Omega^{(k)}\right\}_{k \geq 0}$ be a sequence of matrices with nonnegative integer entries. We put

$$
\Omega^{(k)}=\left(\omega_{i j}^{(k)}\right) \quad \text { and } \quad \Omega^{(k)} z=\left(z_{1}^{(k)}, \ldots, z_{n}^{(k)}\right) .
$$

For $\lambda=\left(\lambda_{1}, \ldots, \lambda_{n}\right)$, we define $z^{\lambda}=z_{1}^{\lambda_{1}} \ldots z_{n}^{\lambda_{n}}$ and $|\lambda|=\lambda_{1}+\ldots+\lambda_{n}$. Let $\left\{f_{1}^{(k)}(z)\right\}_{k \geq 0}, \ldots,\left\{f_{m}^{(k)}(z)\right\}_{k \geq 0}$ be sequences of power series in $K\left[\left[z_{1}, \ldots, z_{n}\right]\right]$. Let $\chi=\left(z_{1}, \ldots, z_{n}\right)$ be the ideal generated by $z_{1}, \ldots, z_{n}$ in $K\left[\left[z_{1}, \ldots, z_{n}\right]\right]$. We assume

$$
f_{i}^{(k)} \rightarrow f_{i} \quad(k \rightarrow \infty), \quad i=1, \ldots, m,
$$

under the topology defined by $\chi$. In what follows, $c_{1}, c_{2}, \ldots$ denote positive constants independent of $k$.

THEOREM 2. Suppose that the coefficients of $f_{i}^{(k)}$ are in a finite set $S \subset K$ for all $i$ and $k$. If $\alpha=\left(\alpha_{1}, \ldots, \alpha_{n}\right) \in K^{n}, 0<\left|\alpha_{i}\right|<1, i=1, \ldots, n$, and the following three properties are satisfied, then $f_{1}^{(0)}(\alpha), \ldots, f_{m}^{(0)}(\alpha)$ are algebraically independent.

(I) There exists a sequence $\left\{r_{k}\right\}_{k \geq 0}$ of positive numbers such that

$$
\lim _{k \rightarrow \infty} r_{k}=\infty, \quad \omega_{i j}^{(k)} \leq c_{1} r_{k}, \quad \log \left|\alpha_{i}^{(k)}\right| \leq-c_{2} r_{k} .
$$

(II) If we put

$$
f_{i}^{(0)}(\alpha)=f_{i}^{(k)}\left(\Omega^{(k)} \alpha\right)+b_{i}^{(k)},
$$

then $b_{i}^{(k)} \in K$ and

$$
\log \left\|b_{i}^{(k)}\right\| \leq c_{3} r_{k} .
$$

(III) For any power series $F(z)$ represented as a polynomial in $z_{1}, \ldots, z_{n}$, $f_{1}, \ldots, f_{m}$ with complex coefficients,

$$
F(z)=\sum_{\lambda, \mu=\left(\mu_{1}, \ldots, \mu_{m}\right)} a_{\lambda \mu} z^{\lambda} f_{1}(z)^{\mu_{1}} \ldots f_{m}(z)^{\mu_{m}},
$$

where $a_{\lambda \mu}$ are not all zero, there exists $\lambda_{0} \in\left(\mathbb{N}_{0}\right)^{n}$ such that if $k$ is sufficiently large, then

$$
\left|F\left(\Omega^{(k)} \alpha\right)\right| \geq c_{4}\left|\left(\Omega^{(k)} \alpha\right)^{\lambda_{0}}\right| .
$$

Proof of Theorem 2. The following lemma is easy to prove. 
LeMma 1. Let $f(z)=\sum_{\lambda_{1}, \ldots, \lambda_{n}} c_{\lambda_{1} \ldots \lambda_{n}} z_{1}^{\lambda_{1}} \ldots z_{n}^{\lambda_{n}} \in \mathbb{C}\left[\left[z_{1}, \ldots, z_{n}\right]\right]$ converge around the origin. If $z$ is sufficiently close to the origin, then

$$
\sum_{|\lambda| \geq H}\left|c_{\lambda_{1} \ldots \lambda_{n}}\right| \cdot\left|z_{1}\right|^{\lambda_{1}} \ldots\left|z_{n}\right|^{\lambda_{n}} \leq \gamma^{H+1} \max _{i}\left|z_{i}\right|^{H},
$$

where $\gamma$ is a positive constant depending on $f(z)$.

LEMMA 2. (i) If $f_{i}^{(k)}-f_{i} \in \chi^{H}$, then

$$
\left|f_{i}^{(k)}\left(\Omega^{(k)} \alpha\right)-f_{i}\left(\Omega^{(k)} \alpha\right)\right| \leq c_{5}^{H+1} e^{-c_{2} r_{k} H} .
$$

(ii) For F(z) in (III) we put

$$
F^{(k)}(z)=\sum_{\lambda, \mu=\left(\mu_{1}, \ldots, \mu_{m}\right)} a_{\lambda \mu} z^{\lambda} f_{1}^{(k)}(z)^{\mu_{1}} \ldots f_{m}^{(k)}(z)^{\mu_{m}} .
$$

Then $F^{(k)}\left(\Omega^{(k)} \alpha\right) \neq 0$ if $k$ is sufficiently large.

Proof. The assumption (I) and Lemma 1 imply (i). We choose a large $H$ satisfying

$$
e^{-c_{2} H}<\left(\prod_{i=1}^{n}\left|\alpha_{i}\right|\right)^{c_{1}\left|\lambda_{0}\right|}
$$

Using (i) we have

$$
\left|F^{(k)}\left(\Omega^{(k)} \alpha\right)-F\left(\Omega^{(k)} \alpha\right)\right| \leq c_{6} e^{-c_{2} H r_{k}} \leq \frac{1}{2} c_{4}\left(\prod_{i=1}^{n}\left|\alpha_{i}\right|\right)^{c_{1}\left|\lambda_{0}\right| r_{k}}
$$

if $k$ is sufficiently large. On the other hand, by (I) and (III),

$$
\left|F\left(\Omega^{(k)} \alpha\right)\right| \geq c_{4}\left|\left(\Omega^{(k)} \alpha\right)^{\lambda_{0}}\right| \geq c_{4}\left(\prod_{i=1}^{n}\left|\alpha_{i}\right|\right)^{c_{1}\left|\lambda_{0}\right| r_{k}} .
$$

This implies the lemma.

We assume $f_{1}^{(0)}(\alpha), \ldots, f_{m}^{(0)}(\alpha)$ are algebraically dependent and deduce a contradiction. There exist a positive integer $L$ and integers $\tau_{\mu}$, not all zero, for $\mu=\left(\mu_{1}, \ldots, \mu_{m}\right)$ with $0 \leq \mu_{i} \leq L$ such that

$$
\sum_{\mu} \tau_{\mu} f_{1}^{(0)}(\alpha)^{\mu_{1}} \ldots f_{m}^{(0)}(\alpha)^{\mu_{m}}=0 .
$$

Let $w_{1}, \ldots, w_{m}, y_{1}, \ldots, y_{m}$ and $t_{\mu}\left(\mu=\left(\mu_{1}, \ldots, \mu_{m}\right), 0 \leq \mu_{i} \leq L\right)$ be variables and put

$$
\begin{aligned}
F^{(k)}(z ; t) & =\sum_{\mu} t_{\mu} f_{1}^{(k)}(z)^{\mu_{1}} \ldots f_{m}^{(k)}(z)^{\mu_{m}} \\
F(z ; t) & =\sum_{\mu} t_{\mu} f_{1}(z)^{\mu_{1}} \ldots f_{m}(z)^{\mu_{m}}
\end{aligned}
$$


and

$$
\sum_{\mu} t_{\mu}\left(w_{1}+y_{1}\right)^{\mu_{1}} \ldots\left(w_{m}+y_{m}\right)^{\mu_{m}}=\sum_{\mu} T_{\mu}(t ; y) w_{1}^{\mu_{1}} \ldots w_{m}^{\mu_{m}} .
$$

Then we obtain

$$
\begin{aligned}
0 & =F^{(0)}(\alpha ; \tau)=\sum_{\mu} \tau_{\mu}\left(f_{1}^{(k)}\left(\Omega^{(k)} \alpha\right)+b_{1}^{(k)}\right)^{\mu_{1}} \ldots\left(f_{m}^{(k)}\left(\Omega^{(k)} \alpha\right)+b_{m}^{(k)}\right)^{\mu_{m}} \\
& =\sum_{\mu} T_{\mu}\left(\tau ; b^{(k)}\right) f_{1}^{(k)}\left(\Omega^{(k)} \alpha\right)^{\mu_{1}} \ldots f_{m}^{(k)}\left(\Omega^{(k)} \alpha\right)^{\mu_{m}} \\
& =F^{(k)}\left(\Omega^{(k)} \alpha ; T\left(\tau ; b^{(k)}\right)\right) .
\end{aligned}
$$

We put $R=K[t]=K\left[\left\{t_{\mu}\right\}_{\mu=\left(\mu_{1}, \ldots, \mu_{m}\right), 0 \leq \mu_{i} \leq L}\right]$ and

$$
V(\tau)=\{Q(t) \in R \mid Q(T(\tau ; y))=0\} .
$$

Then $V(\tau)$ is a prime ideal of $R$.

Definition. For $P(z ; t)=\sum_{\lambda} P_{\lambda}(t) z^{\lambda} \in R\left[\left[z_{1}, \ldots, z_{n}\right]\right]$, we define

$$
\text { index } P(z ; t)=\min \left\{|\lambda| \mid P_{\lambda} \notin V(\tau)\right\} \text {. }
$$

If $P_{\lambda}(t) \in V(\tau)$ for any $\lambda$, then we define index $P(z ; t)=\infty$.

Since $R / V(\tau)$ is an integral domain, we have

$$
\text { index } P_{1}(z ; t) P_{2}(z ; t)=\operatorname{index} P_{1}(z ; t)+\operatorname{index} P_{2}(z ; t) \text {. }
$$

LEMMA 3. The following two properties are equivalent for any $P(z ; t) \in$ $R[z]$.

(i) $P\left(\Omega^{(k)} \alpha ; T\left(\tau ; b^{(k)}\right)\right)=0$ for all large $k$.

(ii) index $P(z ; t)=\infty$.

Proof. We put

$$
P(z ; t)=\sum_{\lambda} Q_{\lambda}(t) z^{\lambda}, \quad Q_{\lambda}(t) \in R
$$

and

$$
Q_{\lambda}\left(T\left(\tau ; f^{(0)}(\alpha)-w\right)\right)=\sum_{\mu} a_{\lambda \mu} w_{1}^{\mu_{1}} \ldots w_{m}^{\mu_{m}} .
$$

We assume (i). Since $b_{i}^{(k)}=f_{i}^{(0)}(\alpha)-f_{i}^{(k)}\left(\Omega^{(k)} \alpha\right)$, we have

$$
\begin{aligned}
0 & =P\left(\Omega^{(k)} \alpha ; T\left(\tau ; b^{(k)}\right)\right) \\
& =\sum_{\lambda} \sum_{\mu} a_{\lambda \mu}\left(\Omega^{(k)} \alpha\right)^{\lambda} f_{1}^{(k)}\left(\Omega^{(k)} \alpha\right)^{\mu_{1}} \ldots f_{m}^{(k)}\left(\Omega^{(k)} \alpha\right)^{\mu_{m}},
\end{aligned}
$$

for all large $k$. Lemma 2 implies $a_{\lambda \mu}=0$ for all $\lambda, \mu$. Hence

$$
Q_{\lambda}\left(T\left(\tau ; f^{(0)}(\alpha)-w\right)\right)=0 .
$$


Since $w_{1}, \ldots, w_{m}$ are variables, $Q_{\lambda}(T(\tau ; y))=0$, which implies (ii). The opposite is trivial.

LEMma 4. index $F(z ; t)<\infty$.

Proof. By the property (III), there exists $k_{0}$ such that $F\left(\Omega^{\left(k_{0}\right)} \alpha ; \tau\right) \neq 0$. If index $F(z ; t)=\infty$, then

$$
F(z ; t)=\sum_{\lambda} P_{\lambda}(t) z^{\lambda}, \quad P_{\lambda}(t) \in V(\tau) .
$$

Noting $T_{\mu}(\tau ; 0)=\tau_{\mu}$, we have

$$
F\left(\Omega^{\left(k_{0}\right)} \alpha ; \tau\right)=\sum_{\lambda} P_{\lambda}(\tau)\left(\Omega^{\left(k_{0}\right)} \alpha\right)^{\lambda}=0,
$$

which is a contradiction.

For a positive integer $p$, we define

$$
\begin{aligned}
R(p) & =\left\{g(t) \in R \mid \operatorname{deg}_{t_{\mu}} g(t) \leq p\right\}, \\
\overline{R(p)} & =R(p) / R(p) \cap V(\tau), \\
d(p) & =\operatorname{dim}_{K} \overline{R(p)} .
\end{aligned}
$$

Lemma 5. $d(2 p) \leq 2^{(L+1)^{m}} d(p)$.

Proof. If $P(t) \in R(2 p)$, it can be expressed as

$$
P(t)=\sum_{\varepsilon} Q_{\varepsilon}(t) \prod_{\mu} t_{\mu}^{\varepsilon(\mu) p},
$$

where $Q_{\varepsilon}(t) \in R(p), \varepsilon$ is a mapping from the set of $\mu$ to $\{0,1\}$ and the sum is taken over all such mappings. If $\left\{\overline{Q_{1}(t)}, \ldots, \overline{Q_{d(p)}(t)}\right\}$ is a base of $\overline{R(p)}$, then the set

$$
\left\{\overline{Q_{i}(t) \prod_{\mu} t_{\mu}^{\varepsilon(\mu) p}}\right\}_{1 \leq i \leq d(p), \varepsilon}
$$

generates $\overline{R(2 p)}$ and the lemma is proved.

Lemma 6. Let $p$ be a sufficiently large integer. Then there exist polynomials $P_{0}(z ; t), \ldots, P_{p}(z ; t) \in K[z ; t]$ with degree at most $p$ in each variable such that the following properties are satisfied.

(i) index $P_{0}(z ; t)<\infty$.

(ii) If we put $E_{p}(z ; t)=\sum_{h=0}^{p} P_{h}(z ; t) F(z ; t)^{h}$, then

$$
\text { index } E_{p}(z ; t) \geq c_{7}(p+1)^{1+1 / n} .
$$

Proof. If we express

$$
P_{h}(z ; t)=\sum_{\lambda} P_{h \lambda}(t) z^{\lambda}, \quad h=0, \ldots, p,
$$




$$
F(z ; t)^{h}=\sum_{\lambda} Q_{h \lambda}(t) z^{\lambda}, \quad h=0, \ldots, p
$$

then

$$
\sum_{h=0}^{p} P_{h}(z ; t) F(z ; t)^{h}=\sum_{\nu}\left(\sum_{h, \lambda, \mu, \lambda+\mu=\nu} P_{h \lambda}(t) Q_{h \mu}(t)\right) z^{\nu} .
$$

We will choose $P_{h \lambda}(t)$ satisfying

$$
\sum_{h, \lambda, \mu, \lambda+\mu=\nu} \overline{P_{h \lambda}(t)} \overline{Q_{h \mu}(t)}=\overline{0} \quad \text { in } \overline{R(2 p)},
$$

for any $\nu=\left(\nu_{1}, \ldots, \nu_{n}\right)\left(\nu_{i} \leq J-1\right)$, where $J$ will be defined below. We define a linear map from $\overline{R(p)}(p+1)^{n+1}$ to $\overline{R(2 p)} J^{n}$ by

$$
\left(\overline{P_{h \lambda}(t)}\right)_{h, \lambda} \mapsto\left(\sum_{h, \lambda, \mu, \lambda+\mu=\nu} \overline{P_{h \lambda}(t)} \overline{Q_{h \mu}(t)}\right)_{\nu}
$$

Since

$$
\operatorname{dim}_{K} \overline{R(p)}^{(p+1)^{n+1}}=d(p)(p+1)^{n+1}, \quad \operatorname{dim}_{K} \overline{R(2 p)}^{J^{n}}=d(2 p) J^{n},
$$

if $d(p)(p+1)^{n+1}>d(2 p) J^{n}$, then there is a nontrivial solution $\left(\overline{P_{h \lambda}(t)}\right)_{h, \lambda}$. By Lemma $5, J=\left[2^{-(L+1)^{m} / n}(p+1)^{1+1 / n}\right]-1$ satisfies the inequality and

$$
\operatorname{index}\left(\sum_{h=0}^{p} P_{h}(z ; t) F(z ; t)^{h}\right) \geq J \geq c_{8}(p+1)^{1+1 / n} .
$$

If index $P_{0}(z ; t)<\infty$, the proof is complete. Otherwise, we set

$$
r=\min \left\{h \mid \operatorname{index} P_{h}(z ; t)<\infty\right\}, \quad E_{p}(z ; t)=\sum_{h=r}^{p} P_{h}(z ; t) F(z ; t)^{h-r} .
$$

Since index $E_{p}(z ; t) F(z ; t)^{r} \geq J$, we have

$$
\text { index } E_{p}(z ; t) \geq J-r \operatorname{index} F(z ; t) \geq c_{7}(p+1)^{1+1 / n} \text {. }
$$

Now we can complete the proof of Theorem 2. Let index $E_{p}(z ; t)=I$ and $\gamma_{1}, \gamma_{2}, \ldots$ denote positive constants depending on $E_{p}(z ; t)$. Let $k \geq \gamma_{1}$, where $\gamma_{1}$ will be determined below. Let

$$
E_{p}(z ; t)=\sum_{\nu} g_{\nu}(z) t^{\nu}, \quad g_{\nu}(z)=\sum_{\lambda} g_{\nu \lambda} z^{\lambda} .
$$

Then $g_{\nu}(z)$ converges in the $n$-polydisc with radius 1 around the origin. Since

$$
\lim _{k \rightarrow \infty} f_{i}^{(k)}\left(\Omega^{(k)} \alpha\right)=f_{i}(0),
$$

we have

$$
\left|b_{i}^{(k)}\right|,\left|T_{\mu}\left(\tau ; b^{(k)}\right)\right| \leq c_{9} .
$$


Thus by Lemma 1 ,

$$
\begin{aligned}
\left|E_{p}\left(\Omega^{(k)} \alpha ; T\left(\tau ; b^{(k)}\right)\right)\right| & \leq \sum_{\nu}\left(\sum_{|\lambda| \geq I}\left|g_{\nu \lambda}\right| \cdot\left|\left(\Omega^{(k)} \alpha\right)^{\lambda}\right|\right)\left|T\left(\tau ; b^{(k)}\right)^{\nu}\right| \\
& \leq \gamma_{2} \max _{i}\left|\alpha_{i}^{(k)}\right|^{I} .
\end{aligned}
$$

We choose a positive number $\theta$ with $e^{-c_{2} c_{7}}<\theta<1$. By the property (I) we have

$$
\left|E_{p}\left(\Omega^{(k)} \alpha ; T\left(\tau ; b^{(k)}\right)\right)\right| \leq \frac{1}{2} \theta^{r_{k}(p+1)^{1+1 / n}} .
$$

We put

$$
E_{p}^{(k)}(z ; t)=\sum_{h=0}^{p} P_{h}(z ; t) F^{(k)}(z ; t)^{h},
$$

and choose a large $H$ satisfying

$$
e^{-c_{2} H} \leq \theta \cdot \theta^{(p+1)^{1+1 / n}} .
$$

If $f_{i}^{(k)}-f_{i} \in \chi^{H}$, by Lemma 2(i) we have

$$
\left|E_{p}\left(\Omega^{(k)} \alpha ; T\left(\tau ; b^{(k)}\right)\right)-E_{p}^{(k)}\left(\Omega^{(k)} \alpha ; T\left(\tau ; b^{(k)}\right)\right)\right| \leq \gamma_{3} e^{-c_{2} H r_{k}} .
$$

Then

$$
\left|E_{p}^{(k)}\left(\Omega^{(k)} \alpha ; T\left(\tau ; b^{(k)}\right)\right)\right| \leq \gamma_{3} e^{-c_{2} H r_{k}}+\frac{1}{2} \theta^{r_{k}(p+1)^{1+1 / n}} \leq \theta^{r_{k}(p+1)^{1+1 / n}} .
$$

On the other hand,

$$
E_{p}^{(k)}\left(\Omega^{(k)} \alpha ; T\left(\tau ; b^{(k)}\right)\right)=P_{0}\left(\Omega^{(k)} \alpha ; T\left(\tau ; b^{(k)}\right)\right)=\text { (say) } \beta_{k} \in K .
$$

By the properties (I) and (II), we easily see $\left\|\beta_{k}\right\| \leq c_{10}^{r_{k} p}$. By the fact that index $P_{0}(z ; t)<\infty$, there are infinitely many $k$ with $\beta_{k} \neq 0$. For such $k$, we have $r_{k}(p+1)^{1+1 / n} \log \theta \geq \log \left|\beta_{k}\right| \geq-2[K: \mathbb{Q}] \log \left\|\beta_{k}\right\| \geq-2[K: \mathbb{Q}] r_{k} p \log c_{10}$.

Dividing both sides by $r_{k}(p+1)^{1+1 / n}$ and letting $p$ tend to $\infty$, we obtain $\log \theta \geq 0$, a contradiction.

3. Proof of Theorem 1. The following lemma is proved in a similar way to the proof of Lemma A.1 in Masser [1].

Lemma 7. Let $b_{1}>\ldots>b_{n} \geq 2$ be pairwise multiplicatively independent integers. Let $\theta=\log b_{1}$ and $\theta_{i}=\theta / \log b_{i}$. Suppose that for each $\alpha$ in a finite set $A$ we are given real numbers $\lambda_{1 \alpha}, \ldots, \lambda_{n \alpha}$ not all zero, and define the sequence

$$
S_{\alpha}(k)=\sum_{i=1}^{n} \lambda_{i \alpha} b_{i}^{\left[\theta_{i} k\right]}, \quad k=0,1,2, \ldots
$$


If $\left\{k_{l}\right\}_{l \geq 1}$ is an increasing sequence of positive integers with $\left\{k_{l+1}-k_{l}\right\}_{l \geq 1}$ bounded, then there exists $\delta>0$ such that

$$
R(\delta)=\left\{k_{l}\left|\min _{\alpha}\right| S_{\alpha}\left(k_{l}\right) \mid \geq \delta e^{\theta k_{l}}\right\}=\left\{m_{l}\right\}_{l \geq 1}, \quad m_{l}<m_{l+1},
$$

is an infinite set and $\left\{m_{l+1}-m_{l}\right\}_{l \geq 1}$ is bounded.

Proof. Let $k_{l+1}-k_{l} \leq K, l \geq 1$. We prove the lemma by induction on $n$. If $n=1$, then $\left\{m_{l}\right\}_{l \geq 1}=\left\{k_{l}\right\}_{l \geq 1}$ is the required sequence. Assume that we have proved the result with $n$ replaced by $n-1$ for some $n \geq 2$ and the result is not true for $n$. Then for any $\delta>0$ and any positive integer $M$ there is $k_{l}$ such that for $k=k_{l}, k_{l+1}, \ldots, k_{l+M}$ we have

$$
S(k)=\min _{\alpha}\left|S_{\alpha}(k)\right|<\delta e^{\theta k} .
$$

We may assume that for each $\alpha \in A$ the numbers $\lambda_{1 \alpha}, \ldots, \lambda_{n-1, \alpha}$ are not all zero. Let $L=\left(\max _{i} \theta_{i}\right)|A| K+1$ and

$$
J=\left\{\left(p_{1}, \ldots, p_{n}, q_{1}, \ldots, q_{n}\right) \mid 0 \leq p_{i}, q_{i} \leq L, p_{n} \neq q_{n}\right\} .
$$

We take $B=A \times J$ and for each $\beta=\left(\alpha, p_{1}, \ldots, p_{n}, q_{1}, \ldots, q_{n}\right) \in B$ we define

$$
\mu_{i \beta}=\lambda_{i \alpha}\left(b_{n}^{q_{n}} b_{i}^{p_{i}}-b_{n}^{p_{n}} b_{i}^{q_{i}}\right), \quad 1 \leq i \leq n-1 .
$$

Since $p_{n} \neq q_{n}$, the pairwise multiplicative independence shows that $\mu_{1 \beta}, \ldots$, $\mu_{n-1, \beta}$ are not all zero. We define

$$
T_{\beta}(k)=\sum_{i=1}^{n-1} \mu_{i \beta} b_{i}^{\left[\theta_{i} k\right]}, \quad k=0,1, \ldots
$$

For any positive integer $k$ there is $\alpha=\alpha(k) \in A$ such that $S(k)=\left|S_{\alpha}(k)\right|$. By the Box Principle, for any $j$ with $l \leq j \leq l+M-|A|$ there exist $\alpha \in A$ and integers $l_{1}, l_{2}$ such that $j \leq l_{1}<l_{2} \leq j+|A|$ and

$$
S\left(k_{l_{1}}\right)=\left|S_{\alpha}\left(k_{l_{1}}\right)\right|, \quad S\left(k_{l_{2}}\right)=\left|S_{\alpha}\left(k_{l_{2}}\right)\right| .
$$

Put

$$
p_{i}=\left[\theta_{i} k_{l_{1}}\right]-\left[\theta_{i} k_{j}\right], \quad q_{i}=\left[\theta_{i} k_{l_{2}}\right]-\left[\theta_{i} k_{j}\right] .
$$

Then $0 \leq p_{i}, q_{i} \leq L$. Since $\theta_{n}>1$ and $l_{1}<l_{2}$ imply $p_{n}<q_{n}$, we have $\beta=\left(\alpha, p_{1}, \ldots, p_{n}, q_{1}, \ldots, q_{n}\right) \in B$ and

$$
T_{\beta}\left(k_{j}\right)=b_{n}^{q_{n}} S_{\alpha}\left(k_{l_{1}}\right)-b_{n}^{p_{n}} S_{\alpha}\left(k_{l_{2}}\right) .
$$

By the assumption, for $j=l, l+1, \ldots, l+M-|A|$ we have

$$
\left|T_{\beta}\left(k_{j}\right)\right|<c \delta e^{\theta k_{j}},
$$

where $c$ is a positive constant. This contradicts the induction hypothesis.

Lemma 8. Let $b_{1}, \ldots, b_{n}$ be integers as in Lemma 7 . Then there exist an infinite set $\Lambda$ of positive integers, a sequence $\{\delta(l)\}_{l \geq 1}$ of positive numbers 
and a total order in $\left(\mathbb{N}_{0}\right)^{n}$ such that if $\lambda>\mu,|\lambda|,|\mu| \leq l$, then

$$
\sum_{i=1}^{n} \lambda_{i} b_{i}^{\left[\theta_{i} q\right]}-\sum_{i=1}^{n} \mu_{i} b_{i}^{\left[\theta_{i} q\right]} \geq \delta(l) e^{\theta q}
$$

for every sufficiently large $q \in \Lambda$. Moreover any subset of $\left(\mathbb{N}_{0}\right)^{n}$ has the least element.

Proof. We put

$$
A(l)=\left\{(\lambda, \mu)\left|\lambda, \mu \in\left(\mathbb{N}_{0}\right)^{n},\right| \lambda|,| \mu \mid \leq l, \lambda \neq \mu\right\} .
$$

For $(\lambda, \mu) \in A(l)$ we set

$$
S_{(\lambda, \mu)}(q)=\sum_{i=1}^{n}\left(\lambda_{i}-\mu_{i}\right) b_{i}^{\left[\theta_{i} q\right]} .
$$

We inductively define $\delta(l)$ and $\Lambda(l)$ as follows. We put $\Lambda(0)=\mathbb{N}$. By Lemma 7 there exists a positive number $\delta(l)$ such that

$$
\Lambda(l)=\left\{q \in \Lambda(l-1)\left|\min _{(\lambda, \mu) \in A(l)}\right| S_{(\lambda, \mu)}(q) \mid \geq \delta(l) e^{\theta q}\right\}
$$

is an infinite set and the differences of two consecutive elements of $\Lambda(l)$ are bounded. We can choose a sequence $\left\{q_{l}\right\}_{l \geq 1}$ satisfying $q_{l} \in \Lambda(l)$ and $q_{l}<q_{l+1}$. There exists a subsequence $\left\{q_{l}^{(1)}\right\}_{l \geq 1}$ of $\left\{q_{l}\right\}_{l \geq 1}$ such that the signs of $S_{(\lambda, \mu)}\left(q_{l}^{(1)}\right),|\lambda|,|\mu| \leq 1$, are fixed for all $l \geq 1$. There exists a subsequence $\left\{q_{l}^{(2)}\right\}_{l \geq 2}$ of $\left\{q_{l}^{(1)}\right\}_{l \geq 2}$ such that the signs of $S_{(\lambda, \mu)}\left(q_{l}^{(2)}\right),|\lambda|,|\mu| \leq 2$, are fixed for all $l \geq 2$. Continuing this process, we obtain a sequence $\left\{q_{l}^{(m)}\right\}_{l \geq m}$ for every $m \geq 1$. We set

$$
\Lambda=\left\{q_{1}^{(1)}, q_{2}^{(2)}, \ldots, q_{l}^{(l)}, \ldots\right\},
$$

and for $\lambda, \mu \in\left(\mathbb{N}_{0}\right)^{n}$ we define $\lambda>\mu$ if and only if $S_{(\lambda, \mu)}(q)>0$ for all large $q \in \Lambda$. Noting $\Lambda(l) \supset \Lambda(l+1)$ and $q_{l}^{(l)} \in \Lambda(l)$ completes the proof of the first part of the lemma. For the second part, we use the following fact (cf. [2], Lemma 2.6.4): if $S$ is a subset of $\left(\mathbb{N}_{0}\right)^{n}$, then there is a finite subset $T$ of $S$ such that for any $\left(\lambda_{1}, \ldots, \lambda_{n}\right) \in S$, there is an element $\left(\mu_{1}, \ldots, \mu_{n}\right) \in T$ with $\mu_{i} \leq \lambda_{i}, i=1, \ldots, n$. If $\mu$ is the least element of $T$, we can easily see it is also the least element of $S$.

Lemma 9. Let $d \geq 2$ and

$$
f_{j}(z)=\sum_{h=0}^{\infty} s_{j, j h} z^{d^{j h}}, \quad s_{j, j h} \in \mathbb{C}^{\times}, j=1,2, \ldots
$$

Then $f_{j}, j=1,2, \ldots$, are algebraically independent over $\mathbb{C}(z)$. 
Proof. If $f_{1}, \ldots, f_{t}$ are algebraically dependent over $\mathbb{C}(z)$, then there exist $a_{\lambda \mu} \in \mathbb{C}$, not all zero, such that

$$
F(z)=\sum_{\lambda, \mu=\left(\mu_{1}, \ldots, \mu_{t}\right)} a_{\lambda \mu} z^{\lambda} f_{1}(z)^{\mu_{1}} \ldots f_{t}(z)^{\mu_{t}}=0 .
$$

We choose a positive integer $l$ satisfying

$$
\max \left\{\lambda \mid a_{\lambda \mu} \neq 0 \text { for some } \mu\right\}<d^{l} .
$$

We define

$$
\begin{aligned}
M & =\max \left\{|\mu| \mid a_{\lambda \mu} \neq 0 \text { for some } \lambda\right\} \geq 1, \\
A & =\left\{\mu|| \mu \mid=M, a_{\lambda \mu} \neq 0 \text { for some } \lambda\right\} .
\end{aligned}
$$

Let $\nu=\left(\nu_{1}, \ldots, \nu_{t}\right)$ be the largest element of $A$ for the lexicographical order and $\kappa$ be the largest integer such that $a_{\kappa \nu} \neq 0$. Letting

$$
\begin{aligned}
p= & \kappa+d^{t ! l+1}+d^{t ! 2 l+1}+\ldots+d^{t ! \nu_{1} l+1}+d^{t !\left(\nu_{1}+1\right) l+2}+\ldots+d^{t !\left(\nu_{1}+\nu_{2}\right) l+2} \\
& +d^{t !\left(\nu_{1}+\ldots+\nu_{t-1}+1\right) l+t}+\ldots+d^{t !\left(\nu_{1}+\ldots+\nu_{t}\right) l+t}
\end{aligned}
$$

we will show that the Taylor coefficient of $z^{p}$ in $F(z)$ is not zero. This contradicts $F(z)=0$ and completes the proof.

The $d$-adic expansion of $p$ has the form

$$
* \ldots * 0 e_{l-1} \ldots e_{0}, \quad 0 \leq e_{i}<d .
$$

If a positive integer $n$ has the $d$-adic expansion

$$
e_{L} \ldots e_{l+1} e_{l} \ldots e_{1} e_{0}, \quad 0 \leq e_{i}<d,
$$

we denote by $w(n)$ the number of nonzero elements among $e_{L}, \ldots, e_{l+1}$. Then $w(p)=\nu_{1}+\ldots+\nu_{t}=M$. For any $a, b \in \mathbb{N}_{0}$, we see $w\left(a+d^{b}\right) \leq w(a)+1$. If $q$ is the degree of a term appearing in the development of

$$
a_{\lambda \mu} z^{\lambda} f_{1}(z)^{\mu_{1}} \ldots f_{t}(z)^{\mu_{t}}
$$

then

$$
\begin{aligned}
q= & \lambda+d^{h_{1}}+\ldots+d^{h_{\mu_{1}}}+d^{2 h_{\mu_{1}+1}}+\ldots+d^{2 h_{\mu_{1}+\mu_{2}}} \\
& +d^{t h_{\mu_{1}+\ldots+\mu_{t-1}+1}}+\ldots+d^{t h_{\mu_{1}+\ldots+\mu_{t}}}
\end{aligned}
$$

where $h_{i} \in \mathbb{N}_{0}$. If $p=q$,

$$
M=w(p)=w(q) \leq w(\lambda)+\mu_{1}+\ldots+\mu_{t}=|\mu| \leq M,
$$

and so $|\mu|=M$. If $w\left(\lambda+d^{j h_{i}}\right)=0, w(q) \leq M-1$. Therefore $w\left(\lambda+d^{j h_{i}}\right)=1$ and so $j h_{i} \geq l+1$ since $\lambda<d^{l}$. Hence we have $\lambda=\kappa$. If $j h_{i}=j^{\prime} h_{i^{\prime}}$ for some $(i, j) \neq\left(i^{\prime}, j^{\prime}\right)$, then $w\left(\lambda+d^{j h_{i}}+d^{j^{\prime} h_{i^{\prime}}}\right)=1$. This implies $w(q) \leq M-1$, a contradiction. Therefore $j h_{i}$ are distinct and

$$
\begin{array}{r}
\left\{t ! l+1, \ldots, t ! \nu_{1} l+1, t !\left(\nu_{1}+1\right) l+2, \ldots, t !\left(\nu_{1}+\nu_{2}\right) l+2,\right. \\
\left.\ldots, t !\left(\nu_{1}+\ldots+\nu_{t-1}+1\right) l+t, \ldots, t !|\nu| l+t\right\}
\end{array}
$$




$$
=\left\{h_{1}, \ldots, h_{\mu_{1}}, 2 h_{\mu_{1}+1}, \ldots, 2 h_{\mu_{1}+\mu_{2}}, \ldots, t h_{\mu_{1}+\ldots+\mu_{t-1}+1}, \ldots, t h_{|\mu|}\right\} .
$$

There are exactly $\nu_{1}$ elements which are not divided by any of $2, \ldots, t$ on both sides above. Therefore $\mu_{1} \geq \nu_{1}$, which implies $\mu_{1}=\nu_{1}$ since $\mu \leq \nu$. Then

$$
\begin{array}{r}
\left\{t !\left(\nu_{1}+1\right) l+2, \ldots, t !\left(\nu_{1}+\nu_{2}\right) l+2, \ldots, t !\left(\nu_{1}+\ldots+\nu_{t-1}+1\right) l+t, \ldots, t !|\nu| l+t\right\} \\
=\left\{2 h_{\mu_{1}+1}, \ldots, 2 h_{\mu_{1}+\mu_{2}}, \ldots, t h_{\mu_{1}+\ldots+\mu_{t-1}+1}, \ldots, t h_{|\mu|}\right\} .
\end{array}
$$

There are exactly $\nu_{2}$ elements which are not divided by any of $3, \ldots, t$ on both sides above. Therefore $\mu_{2} \geq \nu_{2}$, which implies $\mu_{2}=\nu_{2}$ since $\mu \leq \nu$ and $\mu_{1} \leq \nu_{1}$. Continuing, we obtain $\mu=\nu$. Therefore the Taylor coefficient of $z^{p}$ in $F(z)$ is

$$
a_{\kappa \nu} \nu_{1} ! \ldots \nu_{t} ! s_{1, t ! l+1} \ldots s_{1, t ! \nu_{1} l+1} \ldots s_{t, t !\left(\nu_{1}+\ldots+\nu_{t-1}+1\right) l+t} \ldots s_{t, t !|\nu| l+t} \neq 0 .
$$

Proof of Theorem 1. Let

$$
D=\left\{d \in \mathbb{N} \mid d \neq a^{n} \text { for any } a, n \in \mathbb{N}, n>1\right\} .
$$

Then

$$
\mathbb{N} \backslash\{1\}=\bigcup_{d \in D}\left\{d, d^{2}, \ldots\right\} \quad \text { (disjoint union) }
$$

and any two elements of $D$ are multiplicatively independent. Let $d_{1}>\ldots>$ $d_{n}$ be elements of $D, z=\left(z_{1}, \ldots, z_{n}\right)$ and

$$
f_{i j}^{(0)}(z)=\sum_{h=0}^{\infty} \sigma_{i j h} z_{i}^{d_{i}^{j h}}, \quad i=1, \ldots, n, j=1, \ldots, t,
$$

where for any $h, \sigma_{i j h} \in S_{i j}$, which is a finite set of nonzero algebraic numbers. We will show that

$$
\sum_{h=0}^{\infty} \sigma_{i j h} \alpha^{d_{i}^{j h}}, \quad i=1, \ldots, n, j=1, \ldots, t,
$$

are algebraically independent for any algebraic $\alpha$ with $0<|\alpha|<1$. This implies Theorem 1. Put $b_{i}=d_{i}^{t !}, \theta=\log b_{1}, \theta_{i}=\theta / \log b_{i}, i=1, \ldots, n$, and

$$
\Sigma_{q}=\left(\sigma_{i j h}\right)_{i=1, \ldots, n, j=1, \ldots, t, h \geq(t ! / j)\left[\theta_{i} q\right]} \in \prod_{i=1}^{n} \prod_{j=1}^{t} S_{i j}^{\mathbb{N}}
$$

for $q \in \Lambda$ (in Lemma 8). Since the right hand side is a compact set, there exists a converging subsequence $\left\{\Sigma_{q_{k}}\right\}_{k \geq 1}$ of $\left\{\Sigma_{q}\right\}_{q \in \Lambda}$. Let

$$
\lim _{k \rightarrow \infty} \Sigma_{q_{k}}=\left(s_{i j h}\right)_{i=1, \ldots, n, j=1, \ldots, t, h \geq 0}
$$

and

$$
f_{i j}^{(k)}(z)=\sum_{h=(t ! / j)\left[\theta_{i} q_{k}\right]}^{\infty} \sigma_{i j h} z_{i}^{d_{i}^{j\left(h-(t ! / j)\left[\theta_{i} q_{k}\right]\right)}}, \quad f_{i j}(z)=\sum_{h=0}^{\infty} s_{i j h} z_{i}^{d_{i}^{j h}}
$$


Then

$$
\lim _{k \rightarrow \infty} f_{i j}^{(k)}(z)=f_{i j}(z)
$$

We define

$$
\Omega^{(k)}=\left(\begin{array}{ccc}
b_{1}^{\left[\theta_{1} q_{k}\right]} & & 0 \\
& \ddots & \\
0 & & b_{n}^{\left[\theta_{n} q_{k}\right]}
\end{array}\right) .
$$

Then $\Omega^{(k)},\left(\alpha_{1}, \ldots, \alpha_{n}\right)=(\alpha, \ldots, \alpha)$ and $r_{k}=b_{1}^{q_{k}}$ satisfy the assumptions (I) and (II). If the assumption (III) is also satisfied, the assertion follows. Noting $z_{1}, \ldots, z_{n}$ are distinct variables, by Lemma 9 we see

$$
f_{11}, \ldots, f_{1 t}, \ldots, f_{n 1}, \ldots, f_{n t}
$$

are algebraically independent over $\mathbb{C}\left(z_{1}, \ldots, z_{n}\right)$. Let

$$
F(z)=\sum_{\lambda, \mu} a_{\lambda \mu} z^{\lambda} f_{11}^{\mu_{11}} \ldots f_{1 t}^{\mu_{1 t}} \ldots f_{n 1}^{\mu_{n 1}} \ldots f_{n t}^{\mu_{n t}}=\sum_{\lambda \in\left(\mathbb{N}_{0}\right)^{n}} c_{\lambda} z^{\lambda}
$$

and $\lambda_{0}$ be the least element in $\left(\mathbb{N}_{0}\right)^{n}$ in the order defined in Lemma 8 among $\lambda$ with $c_{\lambda} \neq 0$. Let $B=\max \left\{b_{1}, \ldots, b_{n}\right\}$ and $l=\left(\left|\lambda_{0}\right|+1\right) B$. Then

$$
B^{-1} b_{1}^{q_{k}} \leq b_{i}^{-1} b_{1}^{q_{k}}<b_{i}^{\left[\theta_{i} q_{k}\right]} \leq b_{1}^{q_{k}} .
$$

If $k$ is sufficiently large, then by Lemma 1 ,

$$
\sum_{|\lambda| \geq l}\left|c_{\lambda}\right| \cdot|\alpha|^{b_{1}^{\left[\theta_{1} q_{k}\right]} \lambda_{1}} \ldots|\alpha|^{b_{n}^{\left[\theta_{n} q_{k}\right]} \lambda_{n}} \leq \gamma^{l+1}|\alpha|^{b_{1}^{q_{k}}\left(\left|\lambda_{0}\right|+1\right)} .
$$

Since

$$
\lambda_{01} b_{1}^{\left[\theta_{1} q_{k}\right]}+\ldots+\lambda_{0 n} b_{n}^{\left[\theta_{n} q_{k}\right]} \leq\left|\lambda_{0}\right| b_{1}^{q_{k}}
$$

we have

$$
\frac{\left|\sum_{|\lambda| \geq l} c_{\lambda}\left(\Omega^{(k)} \alpha\right)^{\lambda}\right|}{\left|\left(\Omega^{(k)} \alpha\right)^{\lambda_{0}}\right|} \leq \gamma^{l+1}|\alpha|^{b_{1}^{q_{k}}}
$$

if $k$ is sufficiently large. If $|\lambda|<l$ and $\lambda \neq \lambda_{0}$, then by Lemma 8 ,

$$
\frac{\left|c_{\lambda}\left(\Omega^{(k)} \alpha\right)^{\lambda}\right|}{\left|\left(\Omega^{(k)} \alpha\right)^{\lambda_{0}}\right|} \leq\left|c_{\lambda}\right| \cdot|\alpha|^{\delta(l) e^{\theta q_{k}}}
$$

for all large $k$. Therefore

$$
\left|F\left(\Omega^{(k)} \alpha\right) /\left(\Omega^{(k)} \alpha\right)^{\lambda_{0}}-c_{\lambda_{0}}\right| \rightarrow 0 \quad(k \rightarrow \infty) .
$$

This implies (III).

\section{References}

[1] D. Masser, Algebraic independence properties of the Hecke-Mahler series, Quart. J. Math. 50 (1999), 207-230. 
[2] K. Nishioka, Mahler Functions and Transcendence, Lecture Notes in Math. 1631, Springer, 1996.

[3] -, Algebraic independence of reciprocal sums of binary recurrences II, preprint.

Mathematics, Hiyoshi Campus

Keio University

4-1-1 Hiyoshi, Kohoku-ku

Yokohama 223-8521, Japan

E-mail: nishioka@math.hc.keio.ac.jp

Received on 25.10.1999

and in revised form on 3.4.2001 